\title{
Ultraslow light in inhomogeneously broadened media
}

\author{
G. S. Agarwal* and T. N. Dey \\ Department of Physics, Oklahoma State University, Stillwater, Oklahoma 74078, USA
}

(Received 31 October 2005; published 19 April 2006)

\begin{abstract}
We calculate the characteristics of ultraslow light in an inhomogeneously broadened medium. We present analytical and numerical results for the group delay as a function of power of the propagating pulse. We apply these results to explain the recently reported saturation behavior [Baldit et al., Phys. Rev. Lett. 95, 143601 (2005)] of ultraslow light in rare-earth-ion-doped crystal.
\end{abstract}

DOI: 10.1103/PhysRevA.73.043809

PACS number(s): 42.50.Gy, 42.65.-k

The usage of a coherent field to control the optical properties of a medium has led to many remarkable results such as enhanced nonlinear optical effects $[1,2]$, electromagnetically induced transparency (EIT) [3], lasing without inversion [4-6], ultraslow light [7-11], storage and retrieval of optical pulses [12], and many others [13-16]. Most of these effects rely on quantum interferences which are created by the application of a coherent field. The coherent field opens up a new channel for the process under consideration. This interference effect produces the EIT dip or a hole in the absorption profile. The ultraslow light emerges as the EIT dip can be very narrow. It has been realized that in principle one could also use two-level nonlinearities in the presence of a strong pump. For a homogeneously broadened medium a hole can emerge if the transverse and longitudinal relaxation times are quite different. Under these conditions the hole has a width of the order of $T_{1}$ and this is referred to as the effect of coherent population oscillation [17]. Bigelow et al. did experiments in this regime using ruby as the material medium, which can be modeled as a homogeneously broadened system $[18,19]$. Some studies on slow light in inhomogeneously broadened media exist [20,21]. In an earlier paper the present authors considered the case of an inhomogeneously broadened gaseous medium where the Doppler effect is important [21]. We considered the case of saturation absorption spectroscopy. This leads to the well-known hole in the Doppler profile. The width of this hole was of the order of $1 / T_{1}$ which is about two times $1 / T_{2}$. In an inhomogeneously broadened gaseous medium a group index of the order of $10^{3}$ was obtained. The recent experiment of Baldit et al. reports group delays of the order of $1.1 \mathrm{~s}$ in a rare-earthion-doped crystal which has strong inhomogeneous broadening [22]. In this case all the relaxation times are quite different: $T_{1}=8 \mathrm{~ms} ; T_{2}=3 \mu \mathrm{s}$; inhomogeneous linewidth $\Gamma_{i n h}$ $=1.3 \mathrm{GHz}$. The width of the hole is essentially determined by $T_{1}$ and hence one gets very large delays. Baldit et al. did present a theoretical model based on homogeneous broadening of the medium, whereas to obtain agreement with experiments inhomogeneous broadening must be included as mentioned by them [23].

\footnotetext{
*On leave of absence from Physical Research Laboratory, Navrangpura, Ahmedabad 380 009, India.
}

In this paper, we consider a system of inhomogeneously broadened two-level atoms interacting with copropagating pump and probe fields. We use the well-known susceptibility [24] and average it over the inhomogeneous distribution to calculate the group index. We derive a number of analytical results and show how these can be used to understand the experimental results of Baldit et al. For example we show that in the limit of very small detuning of the probe from the pump the group delay varies as $\sqrt{S}$ for large $S$. The group delay also peaks at about $S=0.9$. The value of group delay increases as the detuning $\delta$ increases. We further present detailed numerical results.

In order to understand the experimental results of Baldit et al., we consider a two-level system as shown in Fig. 1.

Here we define all fields as

$$
\vec{E}_{i}(z, t)=\overrightarrow{\mathcal{E}}_{i}(z, t) e^{-i\left(\omega_{i} t-k z\right)}+\text { c.c. } \quad(i=p, c)
$$

where $\overrightarrow{\mathcal{E}}_{i}$ is the slowly varying envelope of the field. The pump field at frequency $\omega_{c}$ and the probe field at frequency $\omega_{p}$ are copropagating through the medium. The linear susceptibility $\chi\left(\omega_{p}\right)$ is obtained by solving the density matrix equations for the two-level system of Fig. 1, that is, by calculating the density matrix element $\rho_{e g}$ to the first order in the probe field but to all orders in the copropagating pump field. The dynamics of population and polarization of the atoms in the two-level configuration are given by

$$
\dot{\rho}_{e e}=-\frac{1}{T_{1}} \rho_{e e}+i\left(G+g e^{-i \delta t}\right) \rho_{g e}-i\left(G^{*}+g^{*} e^{i \delta t}\right) \rho_{e g}
$$

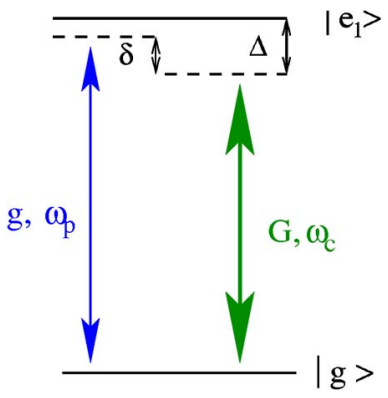

FIG. 1. (Color online) A schematic diagram of two-level atomic system with ground state $|g\rangle$ and excited state $\left|e_{1}\right\rangle$; the pump $\left(\omega_{c}\right)$ and probe $\left(\omega_{p}\right)$ fields are copropagating. 

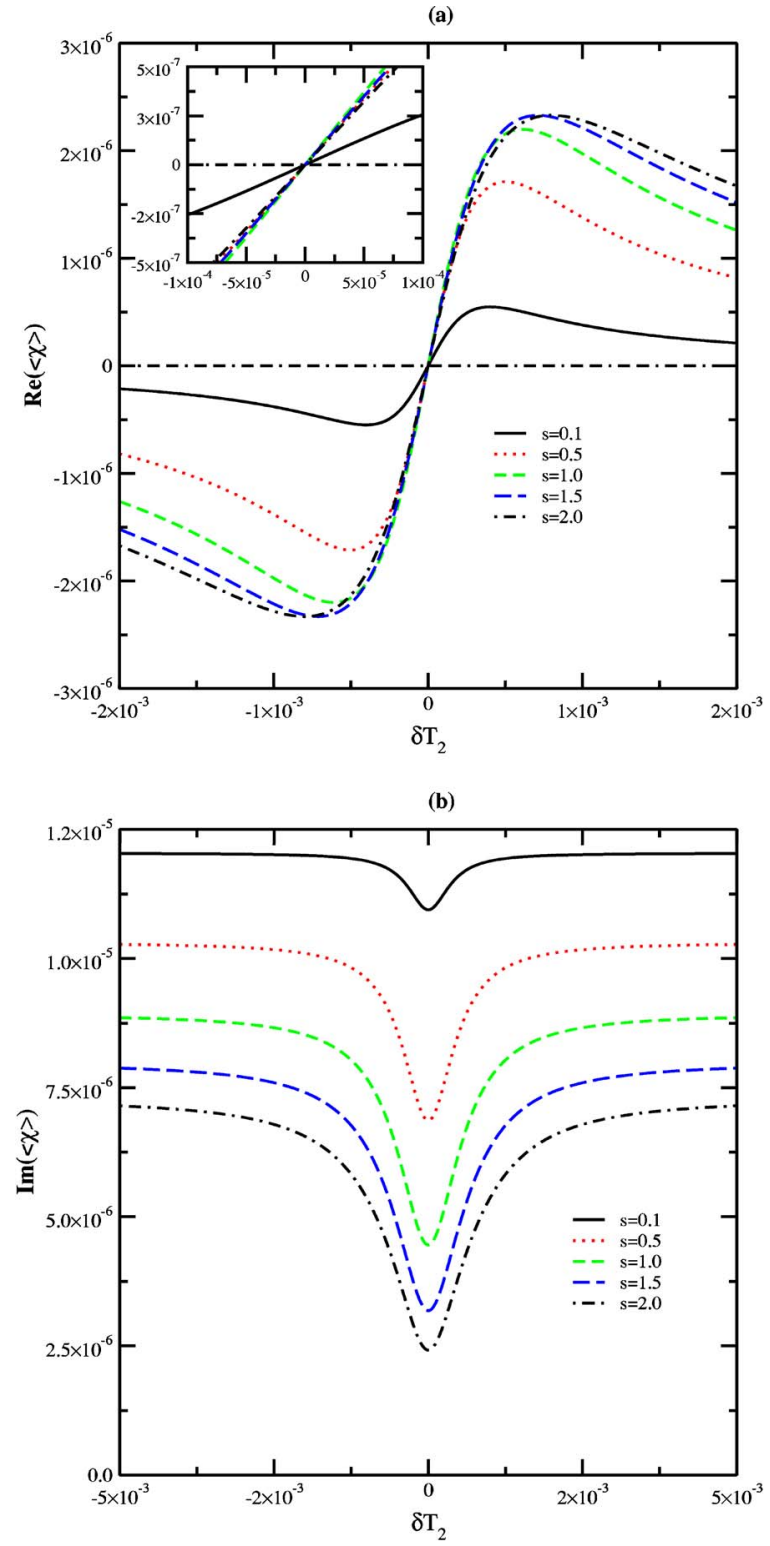

FIG. 2. (Color online) (a) and (b) The real and imaginary parts, respectively, of the susceptibility $\langle\chi\rangle$ at the probe frequency $\omega_{p}$ in the presence of a copropagating control field $G$. The common parameters of the five plots for an erbium-doped crystal are chosen as follows: inhomogeneous absorption coefficient $\alpha_{i n h}=6.5 \mathrm{~cm}^{-1}$; inhomogeneous linewidth $\Gamma_{i n h}=1.3 \mathrm{GHz}$; longitudinal relaxation time $T_{1}=8 \mathrm{~ms}$; transverse relaxation time $T_{2}=3 \mu \mathrm{s}$. The inset shows a zoom of part of the same.

$$
\begin{gathered}
\dot{\rho}_{e g}=-\left(i \Delta+\frac{1}{T_{2}}\right) \rho_{e g}+i\left(G+g e^{-i \delta t}\right)\left(\rho_{g g}-\rho_{e e}\right), \\
\rho_{e e}+\rho_{g g}=1,
\end{gathered}
$$

where $T_{1}$ and $T_{2}$ are the longitudinal and transverse relaxation times, respectively. The density-matrix elements in the original frame are given by $\rho_{e g} e^{-i \omega_{c} t}, \rho_{g g}$, and $\rho_{e e}$. The detunings $\Delta, \delta$ and the Rabi frequencies are defined by

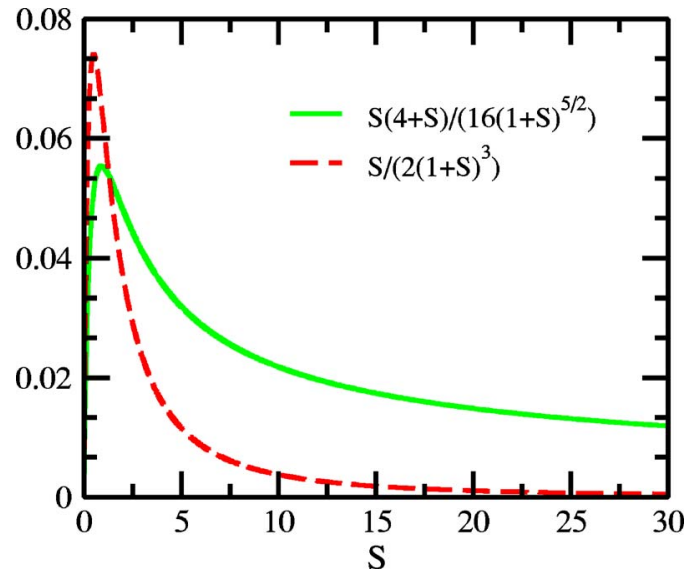

FIG. 3. (Color online) Variation of the term in squared bracket of Eqs. (10) and (11) as a function of intensity of the control field for inhomogeneous and homogeneous cases of a two-level system.

$$
\begin{aligned}
& \Delta=\omega_{e g}-\omega_{c}, \quad \delta=\omega_{p}-\omega_{c}, \\
& 2 G=\frac{2 \vec{d}_{e g} \cdot \overrightarrow{\mathcal{E}}_{c}}{\hbar}, \quad 2 g=\frac{2 \vec{d}_{e g} \cdot \overrightarrow{\mathcal{E}}_{p}}{\hbar},
\end{aligned}
$$

where $\vec{d}_{e g}$ is the dipole matrix element. The susceptibility $\chi$ can be obtained by considering the steady-state solution of Eq. (2) to first order in $g$ and writing the solution as

$$
\rho=\rho^{0}+g e^{-i \delta t} \rho^{+}+g^{*} e^{i \delta t} \rho^{-}+\cdots .
$$

The $e g$ element of $\rho^{+}$will yield the linear susceptibility $\chi$ at the frequency $\omega_{p}$ as can be seen by combining Eqs. (2) and (4):

$$
\begin{aligned}
\chi= & -\frac{n|d|^{2} T_{2}}{\hbar} \frac{1+\Delta^{2} T_{2}^{2}}{\left(1+\Delta^{2} T_{2}^{2}+S\right)\left(\Delta T_{2}+\delta T_{2}+i\right)}(1 \\
& \left.-\frac{S\left(\Delta T_{2}-i\right)^{-1}\left(\delta T_{2}+2 i\right)\left(\delta T_{2}-\Delta T_{2}+i\right)}{2\left(\delta T_{1}+i\right)\left(\delta T_{2}+\Delta T_{2}+i\right)\left(\delta T_{2}-\Delta T_{2}+i\right)-S\left(\delta T_{2}+i\right)}\right),
\end{aligned}
$$

where $n$ is the density of the atoms in the medium. The saturation parameter $S=4|G|^{2} T_{1} T_{2}$ is defined as the ratio of the control field intensity and the saturation intensity. The average response of the susceptibility is given by

$$
\langle\chi\rangle=\frac{2 \sqrt{\ln 2}}{\sqrt{\pi} \Gamma_{i n h}} \int \chi(\Delta) e^{-4 \ln 2\left[\Delta-\left(\bar{\omega}_{e g}-\omega_{c}\right)\right]^{2} / \Gamma_{i n h}^{2} d \Delta,}
$$

where $\bar{\omega}_{e g}$ is the central frequency of the atomic transition $|e\rangle \leftrightarrow|g\rangle$. Here we consider the frequency of the control field $\omega_{c}$ tuned to the line center $\bar{\omega}_{\text {eg }}$. We present the behavior of the real and imaginary parts of the susceptibility as a function of the detuning of the probe field in Fig. 2. The real part of susceptibility gives normal dispersion. It is clear from Fig. 2(a) that the slope of normal dispersion attains its maximum when $S \sim 1$ which leads to ultraslow light. The imaginary part of $\langle\chi\rangle$ exhibits an absorption dip which becomes deeper 
with increase in the intensity of the control field as shown in Fig. 2(b). The spectral width of the absorption dip depends on the intensity of the control field. This dip is associated with coherent population oscillation [17].

In order to compare our treatment with the experimental results of Baldit et al. we need to compute the group index $n_{g}$ which is defined by

$$
n_{g}=1+2 \pi \omega_{p} \frac{\partial}{\partial \omega_{p}} \operatorname{Re}\langle\chi\rangle=1-\frac{\alpha_{i n h} c T_{2}}{2 \pi}\langle D\rangle
$$

where

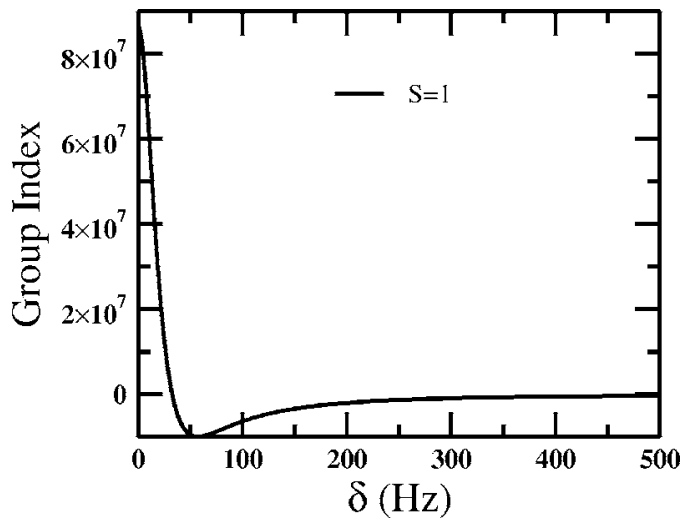

FIG. 5. Group index variation with the detuning of the probe field. The different parameters used in the numerical simulation of Eq. (7) are as follows: $\alpha_{i n h}=6.5 \mathrm{~cm}^{-1}, T_{1}=8 \mathrm{~ms}$, and $T_{2}=3 \mu \mathrm{s}$.

$$
D=\frac{i(\Delta+i)\left[S^{2}+2(\delta f+i)^{2}(i+\delta-\Delta)^{2}(1+i \Delta)+S(i+\delta-\Delta)\left(-i+\delta+2 f\left(i+\delta-i \delta^{2}-\Delta\right)+\Delta\right)\right]}{2\left(1+S+\Delta^{2}\right)\left\{S(\delta+i)-(i+\delta f)\left[(i+\delta)^{2}-\Delta^{2}\right]\right\}^{2}}, \quad f=\frac{T_{1}}{T_{2}}
$$

We denote the averaging over the inhomogeneous profile for the detuning $\Delta$ by \langle\rangle . The unsaturated inhomogeneous absorption coefficient of the two-level atomic system is defined as

$$
\alpha_{i n h}=\frac{4 \pi \omega_{p}}{c}\left\langle\operatorname{Im}[\chi]_{G=0}\right\rangle=\frac{8 \pi^{3 / 2} \omega_{p} n\left|d_{e g}\right|^{2} \sqrt{\ln 2}}{c \hbar \Gamma_{i n h}} .
$$

In the limit of very small detuning of the probe from the pump $\left(\delta \ll 1 / T_{1}\right)$, the analytical expression for the group index for the inhomogeneous case can be expressed as

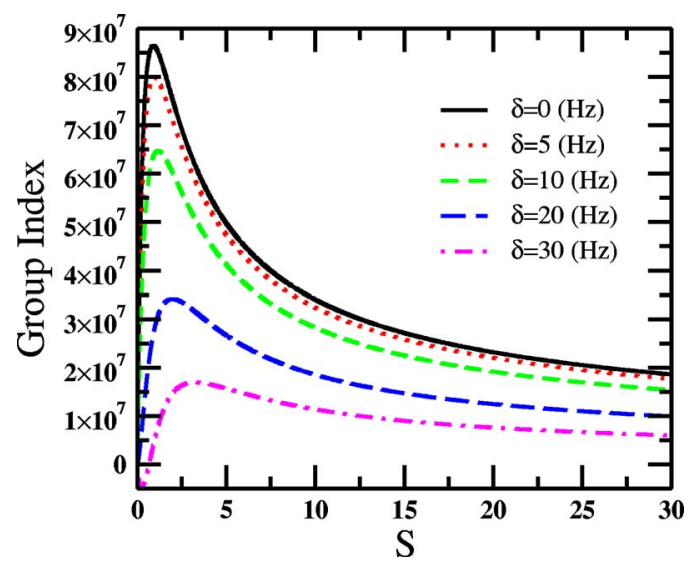

FIG. 4. (Color online) The variation of group index with the saturation parameter $S$. The parameters are chosen as $\alpha_{i n h}=6.5$ $\mathrm{cm}^{-1}, T_{1}=8 \mathrm{~ms}$, and $T_{2}=3 \mu \mathrm{s}$.

$$
n_{g} \cong c \alpha_{i n h} T_{1}\left(\frac{S(4+S)}{16(1+S)^{5 / 2}}\right), \quad \delta \rightarrow 0 .
$$

It is clear from the above expression that the group index varies as $S^{-1 / 2}$ for large values of $S$. The group index attains its maximum value at $S=0.9$. In the case of a homogeneously broadened two level system the group index is given by [22]

$$
n_{g} \cong c \alpha_{h} T_{1}\left(\frac{S}{2(1+S)^{3}}\right), \quad \delta \rightarrow 0,
$$

where $\alpha_{h}=4 \pi \omega_{p} n\left|d_{e g}\right|^{2} T_{2} / c \hbar$ is the homogeneous absorption coefficient. Note that the ratio between inhomogeneous and homogeneous unsaturated absorption coefficients is $\alpha_{h} / \alpha_{i n h}$ $\approx \Gamma_{i n h} T_{2}$. For a homogeneous two-level system the group index varies as $S^{-2}$ at large $S$ and peaks at $S=0.5$. At large $S$, the group index for a two-level system falls much more slowly for an inhomogeneous medium as compared to the homogeneous case as shown in Fig. 3. We thus find an important difference between inhomogeneously and homogeneously broadened two-level systems. Figure 4 shows the variation of group index as a function of the intensity of the control field at different probe detuning. The behavior with respect to the pump power and detunings is in general agreement with the experimental data (cf. for example, at $\delta$ $=10 \mathrm{~Hz}$ the variation of the group index of Fig. 2 of Ref. [22] with our Fig. 4). It is also clear from Fig. 4 that Baldit et al. indeed observed a falloff much slower than $S^{-2}$, which is consistent with our $S^{-1 / 2}$ prediction for $\delta \rightarrow 0$. We also notice from Fig. 4 that the peak of the group index shifts toward higher $S$ as the detuning of the probe field is increased, as observed by them. The maximum of the group index $n_{g}$ $=0.65 \times 10^{8}$ for $\delta=10 \mathrm{~Hz}$ occurs at $S=1.16$ which corre- 
sponds to the group velocity $v_{g}=c / n_{g}=4.61 \mathrm{~m} / \mathrm{s}$ which is higher than what is reported $\left(v_{g}=2.7 \mathrm{~m} / \mathrm{s}\right)$. The possible uncertainty in the value of group velocity is due to its dependence on the inhomogeneous linewidth $\Gamma_{i n h}$, the relaxation times $T_{1}, T_{2}$, and the effective length of the crystal for the experimental conditions $[25,26]$. Further, Fig. 5 shows the variation of the group index $n_{g}$ calculated from Eq. (7) with the detuning of the probe field $\delta$ for saturation parameter $S$ $=1$. We notice from Fig. 5 that the group index changes from positive values to negative values and back to the positive values as the detuning of the probe field is increased. The sign change in group index can be explained with help of Fig. 2(a) wherein the slope of dispersion changes sign as the probe detuning is increased. However Baldit et al. do not report negative group velocities.

In conclusion, we have discussed the characteristics of ultraslow light in an inhomogeneously broadened medium. Our numerical and analytical results enable us to understand the general characteristics of the group index with regard to the pump power and the detuning. We also uncover a region of detuning where negative group velocities occur. We have specifically focussed on the experiment of Baldit et al. However, the results derived here are applicable to any system that can be modeled by an inhomogeneously broadened twolevel system.
[1] S. P. Tewari and G. S. Agarwal, Phys. Rev. Lett. 56, 1811 (1986)

[2] S. E. Harris, J. E. Field, and A. Imamŏglu, Phys. Rev. Lett. 64, 1107 (1990); K.-J. Boller, A. Imamŏglu, and S. E. Harris, ibid. 66, 2593 (1991); J. E. Field, K. H. Hahn, and S. E. Harris, ibid. 67, 3062 (1991).

[3] S. E. Harris, Phys. Today 50(7), 36 (1997).

[4] O. Kocharovskaya and Ya. I. Khanin, JETP Lett. 48, 630 (1988); S E. Harris, Phys. Rev. Lett. 62, 1033 (1989); M. O. Scully, S.-Y. Zhu, and A. Gavrielides, ibid. 62, 2813 (1989).

[5] O. Kocharovskaya, Phys. Rep. 219, 175 (1992); M. D. Lukin, P. R. Hemmer, and M. O. Scully, Adv. At., Mol., Opt. Phys. 42, 347 (2000).

[6] G. S. Agarwal, Phys. Rev. A 44, R28 (1991).

[7] L. V. Hau, S. E. Harris, Z. Dutton, and C. H. Behroozi, Nature (London) 397, 594 (1999).

[8] M. M. Kash, V. A. Sautenkov, A. S. Zibrov, L. Hollberg, G. R. Welch, M. D. Lukin, Y. Rostovtsev, E. S. Fry, and M. O. Scully, Phys. Rev. Lett. 82, 5229 (1999).

[9] D. Budker, D. F. Kimball, S. M. Rochester, and V. V. Yashchuk, Phys. Rev. Lett. 83, 1767 (1999).

[10] A. V. Turukhin, V. S. Sudarshanam, M. S. Shahriar, J. A. Musser, B. S. Ham, and P. R. Hemmer, Phys. Rev. Lett. 88, 023602 (2002).

[11] P. C. Ku, F. Sedgwick, C. J. Chang-Hasnain, P. Palinginis, T. Li, H. Wang, S. W. Chang, and S. L. Chuang, Opt. Lett. 29, 2291 (2004).

[12] D. F. Phillips, A. Fleischhauer, A. Mair, R. L. Walsworth, and M. D. Lukin, Phys. Rev. Lett. 86, 783 (2001).

[13] A. S. Zibrov, M. D. Lukin, L. Hollberg, D. E. Nikonov, M. O. Scully, H. G. Robinson, and V. L. Velichansky, Phys. Rev. Lett. 76, 3935 (1996).
[14] H. Kang and Y. Zhu, Phys. Rev. Lett. 91, 093601 (2003); H. Kang, G. Hernandez, and Y. Zhu, ibid. 93, 073601 (2004).

[15] L. Deng and M. G. Payne, Phys. Rev. Lett. 91, 243902 (2003).

[16] M. D. Stenner, D. J. Gautheir, and M. A. Neifeld, Nature (London) 425, 695 (2003).

[17] S. E. Schwartz and T. Y. Tan, Appl. Phys. Lett. 10, 4 (1967); M. Sargent III, Phys. Rep., Phys. Lett. 43, 223 (1978); R. W. Boyd, M. G. Raymer, P. Narum, and D. J. Harter, Phys. Rev. A 24, 411 (1981); A. D. Wilson-Gordon, ibid. 48, 4639 (1993).

[18] M. S. Bigelow, N. N. Lepeshkin, and R. W. Boyd, Phys. Rev. Lett. 90, 113903 (2003).

[19] M. S. Bigelow, N. N. Lepeshkin, and R. W. Boyd, Science 301, 200 (2003).

[20] O. Kocharovskaya, Y. Rostovtsev, and M. O. Scully, Phys. Rev. Lett. 86, 628 (2001).

[21] G. S. Agarwal and T. N. Dey, Phys. Rev. A 68063816 (2003).

[22] E. Baldit, K. Bencheikh, P. Monnier, J. A. Levenson, and V. Rouget, Phys. Rev. Lett. 95, 143601 (2005).

[23] There are important differences between a Doppler-broadened gaseous medium and a solid-state medium like a rare-earthion-doped crystal. These differences arise from the way the susceptibilities are to be averaged over an inhomogeneous distribution.

[24] B. R. Mollow, Phys. Rev. A 5, 2217 (1972).

[25] K. Bencheikh (private communication) does agree about some uncertainties in the parameters which require new measurements.

[26] We have further checked that a different modeling of the inhomogeneous linewidth, say, in terms of a Lorentzian, yields results for the group index which are almost indistinguishable from those presented in Fig. 4. 